\title{
THE ASIAN DUST AND AEROSOL LIDAR OBSERVATION NET- WORK (AD-NET): STRATEGY AND PROGRESS
}

\author{
Tomoaki Nishizawa, Nobuo Sugimoto, Ichiro Matsui, Atsushi Shimizu, \\ Akiko Higurashi, and Yoshitaka Jin
}

\author{
National Institute for Environmental Studies, 16-2 Onogawa, Tsukuba, 305-0052, Japan, \\ nisizawa@nies.go.jp
}

\begin{abstract}
We have operated a ground-based lidar network AD-Net using dual wavelength $(532,1064 \mathrm{~nm})$ depolarization Mie lidar continuously and observed movement of Asian dust and air pollution aerosols in East Asia since 2001. This lidar network observation contributed to understanding of the occurrence and transport mechanisms of Asian dust, validation of chemical transport models, data assimilation and epidemiologic studies. To better understand the optical and microphysical properties, externally and internally mixing states, and the movements of Asian dust and airpollution aerosols, we go forward with introducing a multi-wavelength Raman lidar to the ADNet and developing a multi-wavelength technique of HSRL in order to evaluate optical concentrations of more aerosol components. We will use this evolving AD-Net for validation of EarthCARE satellite observation and data assimilation to evaluate emissions of air pollution and dust aerosols in East Asia. We go forward with deploying an in-situ instrument polarization optical particle counter (POPC), which can measure size distributions and non-sphericity of aerosols, to several main AD-Net sites and conducting simultaneous observation of POPC and lidar to clarify internally mixed state of Asian dust and air pollution aerosols transported from the Asian continent to Japan.
\end{abstract}

\section{INTRODUCTION}

Since 2001, National Institute for Environmental Studies (NIES) has continuously operated a ground-based lidar network, the Asian Dust and aerosol lidar observation Network (AD-Net), and observed movement of Asian dust and air pollution aerosols in East Asia in cooperation with various research organizations and universities under several research programs [e.g., 1,2]. The ADNet uses a compact two-wavelength (532 and $1064 \mathrm{~nm})$ backscatter $(\beta)$ and one-wavelength $(532 \mathrm{~nm})$ polarization $(\delta)$ Mie lidar system (i.e.,
$2 \beta+1 \delta$ Mie lidar system) and contributed to understanding of the occurrence and transport mechanisms of Asian dust, validation of chemical transport models, and data assimilation study [e.g., 3,4]. Furthermore, concentrations of Asian dust and air pollution aerosols evaluated from the AD-Net observation has been used in epidemiologic studies (e.g., effect on a human body) [5].

To better understand the optical and microphysical properties and the movements of Asian dust and air-pollution aerosols, we go forward with attaining the multichannel of the lidars settled at several main sites in AD-Net, using a Raman lidar technique, a high spectral resolution lidar (HSRL) technique, and their combined techniques. The independent extinction and multichannel measurements can provide useful data for characterizing aerosol optical properties as demonstrated by multi-wavelength Raman lidar measurements in EARLINET [6]. In addition, the independent multichannel measurements are essential for classifying individual aerosol components (e.g., mineral dust, sea-salt, and black carbon) [7]. Derived extinction coefficients, backscatter coefficients, and depolarization ratio for total aerosols at multi-wavelengths and optical properties for individual aerosol components are useful for validations of chemical aerosol transport models and satellite measurements and data assimilation as well as aerosol characterization. HSRL is a highly sensitive method and helps us to measure signals with sufficient signal-to-noise ratio in daytime as well as nighttime. Thus, we have developed a compact and automatically operated two-wavelength $(355,532 \mathrm{~nm})$ HSRL using a injection seeded Nd:YAG laser, a Fabry-Perot etalon interference at $355 \mathrm{~nm}$ and iodine absorption filter at 532nm [12]. We start developing a novel HSRL using a multi-mode Nd:YAG laser and MachZender interferometer to realize a robuster and 
lower-cost multi-wavelength HSRL [8]. To clarify internally mixed state of Asian dust and air pollution aerosols transported from the Asian continent to Japan, we go forward with introduction of an in-situ instrument optical particle counter with a function of depolarization measurement to several main sites of AD-Net $[9,10]$.

In this paper, we report the latest status of the ADNet observation (Section 2), and denote future strategy and ongoing projects of the AD-Net (Section 3).

\section{LATEST STATUS OF AD-NET}

Fig. 1 indicates the locations of AD-NET observation sites. Table 1 summarizes information for the individual observation sites. AD-Net has 20 observation sites in East Asia and is recognized as one of the contributing network of $\mathrm{WMO} / \mathrm{GAW}$. A part of the network is operated as the Asian dust monitoring network of Ministry of the Environment (MOE), Japan, and a part of the network is operated in the radiation network SKYNET [11].

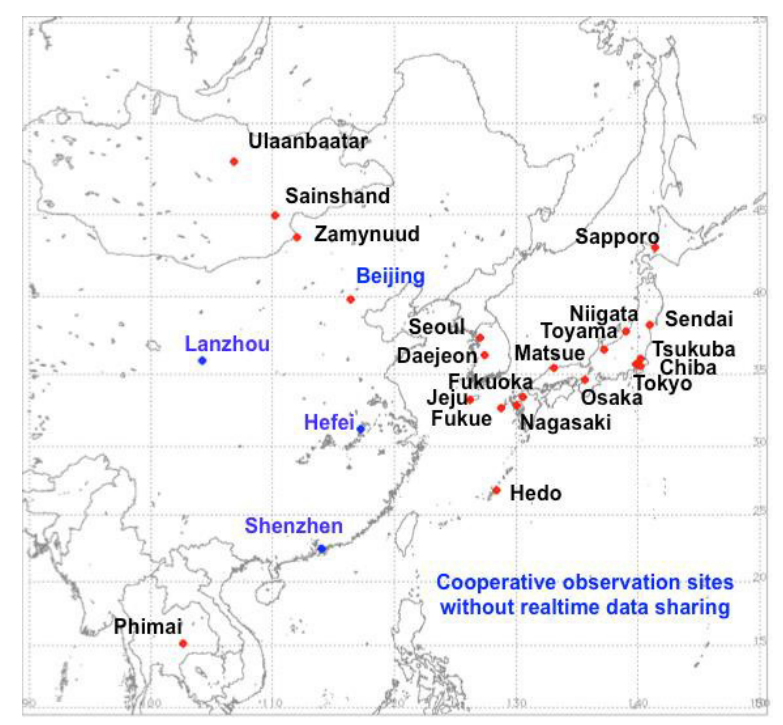

Fig. 1 AD-Net observation sites

\subsection{Standard AD-Net lidars}

Lidars are settled in a container or a building with a glass window on the roof and operated regardless of weather conditions continuously. $2 \beta(532,1064)+1 \delta(532)$ Mie lidars are settled at 13 sites of the AD-Net. A commercial Nd:YAG laser is employed as a light source and emitted into zenith direction in $10 \mathrm{~Hz}$ repetition, $20 \mathrm{~mJ} /$ pulse for both wavelengths. We use a cassegrainian telescope of $20 \mathrm{~cm}$ diameter, two photo multiplier tubes (PMTs) in analogue mode for copol and crosspol signal measurements at $532 \mathrm{~nm}$, an avalanche photo diode (APD) for $1064 \mathrm{~nm}$ signal measurements. They are operated automatically, and the 5-minute averaged lidar profiles with $6 \mathrm{~m}$ vertical resolution are recorded every 15 minutes in the continuous observations. The recorded data are transferred to NIES in real time except for the cooperative stations in China (i.e., Lanzhou, Hefei, and Shenzhen). The observed data are analyzed automatically, and attenuated backscatter coefficients at 532 and $1064 \mathrm{~nm}$, total depolarization ratio at $532 \mathrm{~nm}$, extinction coefficient, backscatter coefficient, and depolarization ratio of aerosols at $532 \mathrm{~nm}$, mixing layer height, and extinction coefficients of mineral dust and spherical aerosols at $532 \mathrm{~nm}$ are distributed in public in quasi real time as the standard product of AD-Net [http://www-lidar.nies.go.jp/AD-Net/]. We use the Fenrald's method with $\mathrm{S} 1=50 \mathrm{sr}$ to estimate aerosol extinction and backscatter coefficients. We then apply the method using the particle depolarization ratio to estimate the dust and spherical aerosol extinction coefficients [1]. It should be noted that the AD-Net standard product are retrieved for all the 20 sites of AD-Net and those are distributed in public.

\section{Table 1 Information for the AD-Net sites}

\begin{tabular}{|c|c|c|c|c|c|c|}
\hline Site & Organization & $\begin{array}{l}\text { Lon. } \\
\text { (E) }\end{array}$ & $\begin{array}{l}\text { Lat. } \\
\text { (N) }\end{array}$ & $\begin{array}{l}\text { Height } \\
\text { (m) }\end{array}$ & $\begin{array}{c}\text { Start of } \\
\text { Observation }\end{array}$ & $\begin{array}{c}\text { Lidar } \\
\text { system }\end{array}$ \\
\hline Tsukuba, Japan ${ }^{-1}$ & NIES & 140.12 & 36.05 & 30 & 1996 & B, D \\
\hline Toyama, Japan ${ }^{-1}$ & MOE/Toyama & 137.10 & 36.70 & 28 & 2004 & A \\
\hline Matsue, Japan & MOE/Shimane & 133.01 & 35.48 & 5 & 2005 & B \\
\hline Nagasaki, Japan & MOE/Nagasaki & 129.98 & 32.94 & 206 & 2001 & A \\
\hline Niigata, Japan & MOE/Niigata & 138.94 & 37.84 & 1 & 2007 & A \\
\hline Tokyo, Japan & $\mathrm{MOE}$ & 139.71 & 35.69 & 42 & 2008 & A \\
\hline Sapporo, Japan ${ }^{* 1}$ & Hokkaido Univ. & 141.33 & 43.06 & 30 & 2003 & A \\
\hline Sendai, Japan" ${ }^{-1}$ & Tohoku Univ. & 140.83 & 38.26 & 154 & 2005 & A \\
\hline Chiba, Japan ${ }^{* 1}$ & Chiba Univ. & 140.12 & 35.62 & 20 & 2007 & A \\
\hline Osaka, Japan & Kinki Univ. & 135.59 & 34.65 & 19 & 2008 & A \\
\hline Fukue, Japan & Chiba Univ. / NIES & 128.68 & 32.75 & 50 & 2002 & B \\
\hline Hedo, Japan ${ }^{-1}$ & NIES & 128.25 & 26.87 & 60 & 2005 & $\mathrm{C}$ \\
\hline Fukuoka, Japan" & Kyushu Univ. & 130.48 & 33.52 & 30 & 2012 & $\mathrm{C}$ \\
\hline Seoul, Japan ${ }^{1}$ & Seoul Nat. Univ. & 126.95 & 37.46 & 116 & 2006 & B \\
\hline Gosan Jeju, Korea & Seoul Nat. Univ. & 126.16 & 33.29 & 35 & 2010 & A \\
\hline Daejeon, Korea & Mokwon Univ. & 127.34 & 36.33 & 80 & 2002 & A \\
\hline Ulaanbaatar, Mongolia & IMH & 106.90 & 47.92 & 1320 & 2007 & A \\
\hline Sainshand, Mongolia & IMH & 110.12 & 44.87 & 937 & 2007 & A \\
\hline Zamyuud, Mongolia & IMH & 111.90 & 43.72 & 962 & 2007 & A \\
\hline Phimai, Thailand"1 & Chulalongkorn Univ. & 102.57 & 15.18 & 212 & 2002 & B \\
\hline
\end{tabular}

\subsection{Introduction of Raman lidar technique}

We have improved the AD-Net Mie lidars at six main sites (i.e., Tsukuba, Fukue, Hedo, Matsue, Seoul, and Phimai) by adding a nitrogen Raman scatter measurement channel since 2009 to better understand aerosol optical properties and realize more advanced aerosol classification [12,13]. The improved lidars (i.e., $1 \alpha+2 \beta+1 \delta$ Mie-Raman lidar) provide extinction $(\alpha)$ at $532 \mathrm{~nm}$, backscat- 
ter $(\beta)$ at 532 and $1064 \mathrm{~nm}$, and depolarization ratio $(\delta)$ at $532 \mathrm{~nm}$. We use a PMT in photon-counting mode for the Raman backscatter measurements. The recorded data are transferred to NIES in real time except for the cooperative station, Beijing in China. The observed data are automatically analyzed [13]. We apply wavelet transform analysis to reduce signal noise; then average the observed data every hour and $120 \mathrm{~m}$ range; and retrieve the $1 \alpha+2 \beta+1 \delta$ data by the conventional direct method using nighttime data observed with appropriate signal-to-noise ratio. To evaluate aerosol components using these $1 \alpha+2 \beta+1 \delta$ data, we developed an algorithm to retrieve extinction coefficients at $532 \mathrm{~nm}$ for four aerosol components: strong light absorption aerosols such as black carbon (BC), nonspherical aerosols such as mineral dust (dust), large-size and spherical aerosols such as sea-salt (sea-salt), and small-size, weak-absorption aerosols such as air-pollution aerosols excepting for black carbon (air pollution) using the derived $1 \alpha+2 \beta+1 \delta$ data $(1 \alpha+2 \beta+1 \delta$ method), and we demonstrated the algorithm performance by applying to the observed data [13]. This method assumes the external mixture of the four aerosol components (i.e., BC, dust, sea-salt, and air pollution) at each layer. Size distributions, refractive indices, particle shapes, and optical properties of the aerosol components are modeled based on previous studies. The vertical profiles of the extinction coefficients at $532 \mathrm{~nm}$ for the four aerosol components are estimated by a look-up table method using their modeled optical properties.

\section{STRATEGY AND ONGOING PROJECT}

\subsection{Introduction of multi-wavelength Raman lidars for more advanced data assimilation}

We deployed a two wavelength Raman lidar at Hedo and Fukuoka sites of AD-Net as well as University of Toyama, Japan (36.70N, 140.12E) and started continuous measurements using the lidars in collaboration with Kyushu university and University of Toyama. The main objective of this study is to estimate extinction coefficients of aerosol components, conduct data assimilation analysis using the retrieved concentrations of aerosol components, and finally evaluate emission of dust and air pollution aerosols and build aerosol assimilated data in each region of East Asia. The multi-wavelength Raman lidar system provides $2 \alpha(355,532)+3 \beta(355,532,1064)+2 \delta(355,532)$ data. The lidar uses a commercial Nd:YAG laser and emit the laser into zenith direction in $10 \mathrm{~Hz}$ repetition and laser energies of 150, 100 and $60 \mathrm{~mJ} /$ pulse for $1064,532,355 \mathrm{~nm}$. We use a cassegrain telescope of $20 \mathrm{~cm}$ diameter, and PMTs for elastic channels at 532 and $355 \mathrm{~nm}$ in analog mode and for nitrogen Raman channels at 607 and $387 \mathrm{~nm}$ in photon-counting mode. Depolarization measurement functions at 355 and $532 \mathrm{~nm}$ are implemented. We will retrieve extinction coefficients of dust, sea-salt, BC, and air pollution aerosols and size distributions of dust, sea-salt, and air pollution aerosols using the $2 \alpha+3 \beta+2 \delta$ data in a similar to the $1 \alpha+2 \beta+1 \delta$ method. This algorithm assumes external mixture of the four aerosol components; the size distributions, refractive indices, and particle shapes and optical properties except for the size distributions of dust, seasalt, and air pollution aerosols are modeled based on previous studies [14].

\subsection{Development of multi-wavelength HSRL for ground-based lidar network observations}

We have developed a compact and automatically operated two-wavelength $(355,532 \mathrm{~nm}) \mathrm{HSRL}$ using a single-longitudinal mode laser, a FabryPerot etalon interference at $355 \mathrm{~nm}$ and iodine absorption filter at 532nm [15]. Using this iodine absorption filter technique for $532 \mathrm{~nm}$ and a nitrogen Raman lidar technique for $355 \mathrm{~nm}$, we constructed a $2 \alpha(355,532)+3 \beta(355,532,1064)+$ $2 \delta(355,532)$ HSRL-Raman lidar at the Tsukuba site of AD-Net and started continuous measurement from 2014 (Table 1). Since the multi-wavelength HSRL system has to fix the laser wavelength to an iodine absorption line and further control the etalon transmittance line to the fixed laser wavelength, and keep controlling the wavelength of the laser and the etalon during continuous measurement, this system has technical complexity and difficulty. To reduce the complexity and difficulty, we start developing a new HSRL technique using a multi-longitudinal mode Nd:YAG laser and Mach-Zender interferometer [8]. The key feature of this system is not to need controlling the laser wavelength though the interferometer has to be controlled. Further, this system is applicable to any laser wavelength, different from HSRL using an iodine absorption filter. In addition, this system is lower-cost since it does 
not use a single-longitudinal mode laser. Thus, we expect that the multi-wavelength HSRL using this developed HSRL technique will be more effective and appropriate for ground-based lidar network observations.

\subsection{Validation observation of the EarthCARE}

An international aerosol, cloud, and radiation observation mission from space "EarthCARE" is ongoing by ESA, JAXA, and NICT. A 355nm high-spectral resolution lidar ATLID as well as a multi spectral imager MSI, a cloud profiling radar $\mathrm{CPR}$, and a broad band radiometer BBR are aboard the EarthCARE satellite [16]. We are developing an algorithm to estimate $1 \alpha+1 \beta+1 \delta$ at $355 \mathrm{~nm}$, mixing layer height, aerosol/cloud discriminator, and extinction coefficients of $\mathrm{BC}$, seasalt, dust, and air pollution aerosols from the ATLID and MSI measurements in collaboration with JAXA to distribute those derived parameters as JAXA level 2 products in public after the EarthCARE will be launched. We are ongoing to introduce multi-wavelength Raman lidars and HSRLs with measurement function at $355 \mathrm{~nm}$ to AD-Net towards the EarthCARE validation observation. It should be noted that the data analysis of the multi-channel lidar with more channels than the ATLID is effective to validate the ATLID-derived extinction coefficients of aerosol components because the algorithm using the multi-channel lidar data needs less assumption and so more accurate (see subsection 3.1).

\subsection{Introduction of polarization optical parti- cle counter (POPC) to study internal mixture of aerosols}

The POPC is an in-situ instrument to measure the forward scattering and the two polarization components of backscattering for single particles at $780 \mathrm{~nm}$ and provides particle size and depolarization ratio for single particles. We conducted simultaneous observations using the POPC and the lidar at the AD-Net site in Seoul and captured the characteristics of pure Asian dust and internally mixed polluted Asian dust; the optical characteristics measured using the lidar were consistent with the POPC measurements [10]. This indicates that the simultaneous measurements of POPC and lidar are effective to clarify and characterize internally mixing state of Asian dust and air pollution aerosols. We will deploy the POPC to the other AD-Net sites.

\section{ACKNOWLEDGEMENT}

This study was partly supported by the Environmental Research and Technology Development Fund of the MOE, Japan (5B-1202), Grant-in-Aid for Scientific Research (25220101) from Japan Society for the Promotion of Science (JSPS), and EarthCARE first algorithm and validation research announcement by JAXA.

\section{REFERENCES}

[1] Shimizu, A., et al., 2004: J. Geophys. Res., 109, D19S17, doi:10.1029/2002JD003253.

[2] Sugimoto, N., et al., 2005: Water, Air, and Soil Pollution: Focus, 5, 145-157.

[3] Yumimoto, K., et al., 2008: Atmos. Chem. Phys., 8, 2869-2884.

[4] Uno, I., et al., 2008: Geophys. Res. Lett., 35, L06803, doi:10.1029/2007GL032329.

[5] Onishi, K., et al., 2012: Atmos. Env., 49, 94102, doi:10.1016/j.atmosenv.2011.12.018.

[6] URL: http://www.earlinet.org/

[7] Nishizawa, T., et al., 2008: Trans. Geosci. Rem. Sens., 41, 1743-54.

[8] Jin, Y., et al., 2015: Proc. $27^{\text {th }}$ ILRC, to be submitted.

[9] Kobayashi, H., et al., 2014: Atmos. Env., http://dx.doi.org/10.1016/j.at-

mosenv.2014.05.006.

[10] Sugimoto, N., et al., 2015: J. Quant. Spec. Rad. Trans., 150, 107-113.

[11]http://atmos.cr.chiba-u.ac.jp/index_atmos.html

[12] Xie, C., et al., 2008: Appl. Opt., 47, 49454951.

[13] Nishizawa, T., et al., 2012: Proc of SPIE, 8526, 8526Q, doi:10.1117/12.977215.

[14] Nishizawa, T., et al., 2008: Proc. 24th

ILRC, 361-364.

[15] Nishizawa, T., et al., 2010: Proc. 25th ILRC, 903-906.

[16] Illingworth, A. J., et al., 2016: BAMS, doi:10.1175/BAMS-D-12-00227.1 